$\sqrt{3}$

J. bio-sci. 20: 57-65, 2012

ISSN 1023-8654

http://www.banglajol.info/index.php/JBS/index

\title{
EVALUATION OF SOME BIOCHEMICAL CHANGES IN THE SERUM OF PHENSEDYL ADDICTS OF GAIBANDHA DISTRICT, BANGLADESH
}

\author{
Md Rashidul Hasan §, Parvez Hassan*, M Abdul Jalil Miah \\ Institute of Biological Sciences, ${ }^{1}$ Department of Chemistry, \\ University of Rajshahi, Rajshahi-6205, Bangladesh
}

\begin{abstract}
Context: Abuse of the drug, Phensedyl like any other drug might exert adverse effects on vital organs of the human body like liver, kidney and heart.

Objectives: To determine the effects of Phensedyl intake on the serum biochemical parameters of the addicts in order to access for damages of vital human organs like liver, kidney and heart.

Materials and Methods: Study population consisted of 127 male Phensedyl addicts within the ages of 18-55 years of defined criteria from Gaibandha district, a Northern part of Bangladesh, during July 2009 to December 2011. Fifty (50) non-drug dependent healthy men of matched age, height, and socioeconomic status were included as controls from the same community. Biochemical parameters analyzed were - Serum creatinine, SGOT, SGPT and Lipid profiles (total serum cholesterol (TC), Serum triglyceride (TG), serum high-density lipoprotein (HDL) and low-density lipoprotein (LDL) cholesterol determined by semiautomatic biochemistry analyzer using commercially available kits

Results: Abuse of Phensedyl appears not to hamper the normal renal and liver function in most of the addicts even after 8 years of Phensedyl intake irrespective of age except in case of 4 abusers. Serum total cholesterol (TC) remained almost unchanged among the addicts abusing Phensedyl for less than 8 years. But very strikingly, addicts taking Phensedyl for more than 8 years had higher trends in serum cholesterol i.e. more than $200 \mathrm{ml} / \mathrm{dl}$. Of the addict's, 44.36\% abusing Phensedyl for less than 8 years had normal triglyceride (TG) values whereas, in $45.66 \%$ addicts abusing the drug for more than 8 years had clinically very significantly elevated triglyceride levels, which was also found to be statistically very significant ( $p$ value $=0.0001$ ), indicating the risk of developing cardiac diseases. Of the total addicts $53.53 \%$ had low levels of HDL cholesterol, which is clinically and statistically found to be very significant ( $p$ value $=0.002$ ). Of the addict's $43 \%$ taking the drug for less than 8 years had normal LDL levels but significantly elevated values were recorded in $34 \%$ of the abusers who had been taking the drug for more than 8 years.

Conclusion: Long time (> 8 years) Phensedyl abusers are at the high risk of developing Brain stroke, Coronary Heart Disease (CHD), Ischemic stroke or transient ischemic attack (TIA) as there is the triad of: Elevated LDL cholesterol, Low HDL cholesterol and elevated Triglyceride.
\end{abstract}

Key words: Addiction, biochemical parameters, Phensedyl, Bangladesh, blood serum.

\section{Introduction}

Drug addiction is a life threatening challenge to the public health. The drug addicts are prone to develop chronic killer diseases like AIDs, cancers, cardiovascular diseases, kidney dysfunction (UNDCP 1997, Varela et al. 1997, WHO 1992, Battjes et al. 1989, Harris and Garret 1972). In Bangladesh, majority of drug addicts are claimed to be suffering from infectious diseases like tuberculosis, hepatitis, respiratory infections, contagious skin diseases (CTC 2000) and STDs and some of them are also inflicted with HIV infection

\footnotetext{
* Corresponding author E-mail: hassanpru1@gmail.com

$\S$ Present Address: Dept. of Biochemistry, Shahid Ziaur Rahman Medical College Hospital, Bogra, Bangladesh.
} 
(ICDDR'B 1999). The abuse (drinking) of an antitussive, Phensedyl syrup has been reported in several countries in South Asia. In India, it is reportedly being used as a cheap substitute for drugs or alcohol. In Nepal, to prevent such abuse, the sale of Phensedyl and other codeine-based cough syrups has been prohibited. Increasing abuse of Phensedyl was reported in Bangladesh. The abuse of Phensedyl in Bangladesh is extremely high possibly because of its easy availability and comparatively lower price. Phensedyl, once available only in $250 \mathrm{ml}$ medicine bottles that were smuggled into Bangladesh out of India, can now be found in large barrels. The codeine phosphate content of some Phensedyl batches found on the illicit markets in Bangladesh and Nepal is much higher than that of the Phensedyl that is licitly available in India (Release of INCB Report for 1995 Regional Update: South Asia).

According to the Government of Bangladesh, Phensedyl has become the main drug of abuse among young people in some areas of the country, including Dhaka. Illicit traffic in and abuse of Phensedyl and other similar cough syrups have created problems in some south-east Asian countries as well (for example Myanmar (Release of INCB Report for 1995 Regional Update: South Asia).

Any drug when abused may exert adverse effects on vital organs of the human body resulting in different degrees of damages to organs like liver, kidney, heart and others. Abuse of the drug Phensedyl, like any other drugs might be associated in development of health problems, different pathophysiological states i.e. various types of diseases. Following the biochemical changes after drug intake may be useful in such assessments.

Although researches on drug addiction and drug abuse have been carried out globally and as well as in Bangladesh, as far as our knowledge goes; no biochemical study appears to be having been done so far in Bangladesh and else where on Phensedyl abuse. The illicit and increasing abuse of the drug Phensedyl in Bangladesh may be associated in developing health problems. To establish the fact, in the present study, several important parameters of biochemical aspect have been performed using the blood samples of the Phensedyl abusers of Gaibandha District, in the Northern region of Bangladesh were the abuse of Phensedyl is reportedly to be very high (Two held with Phensedyl in Gaibandha, The Daily Sun 2010).

\section{Materials and Methods}

The study population consisted of 127 male Phensedyl addicts within the ages of 18-55 years of defined criteria (Male individuals who only took Phensedyl, not multi drug users, belonging to the age group of 18-55 yrs and having no evidence of coronary heart disease (CHD), stroke or other major illnesses) from Gaibandha district, a northern part of Bangladesh, during the period of July 2007 to December 2009. Fifty (50) non-drug dependent (non-users of any tobacco, betel leaves or nuts) healthy men who were similar in age, height, and socioeconomic status were recruited by convenience as controls from the same community.

To conduct the study ethical permission was sought from the Director, Institute of Biological Sciences, University of Rajshahi, Bangladesh. The study subjects were briefed about the objective of the study and a written consent was obtained from each of them. Then blood specimens $(10 \mathrm{ml})$ were collected from each of the subjects by venipuncture, which were used as research tool. Biochemical parameters analyzed include Serum creatinine, SGOT, SGPT and Lipid profiles (total serum cholesterol (TC), Serum triglyceride (TG), serum high-density lipoprotein (HDL) and low-density lipoprotein (LDL) cholesterol.

Estimation of serum creatinine was done by Creatinine liquicolor kit (Human, Germany) by Jaffe - Reaction, Serum cholesterol by enzymatic - colorimetric (CHOD - PAP) method using Cholesterol (Spinreact SA, Spain) kit on TC 84 chemistry Analyzer (Teco Diagnostics, USA) at $505 \mathrm{~nm}$. Serum Triglyceride was determined by enzymatic-colometric GPO - PAP method using Triglyceride GPO liquicolor (Human GMBH, Germany) kit, Serum HDL cholesterol by phosphotungstate / magnesium method (Burstein et al., 
1970) using HDL cholesterol (Spinreact SA, Spain) kit. LDL-Cholesterol was calculated from the estimated serum triglycerides, Total Cholesterol (TC) and HDL-Cholesterol by using the Friedwald's formula (Friedewald et al.1972). Statistical data analysis of was performed using SPSS windows version.

\section{Results}

This study has investigated one hundred twenty seven (127) male Phensedyl addicts of within the ages of 18-45 years. Their serum blood were analyzed for some biochemical parameters of clinical importance and was compared with non-drug dependent control subjects.

Serum creatinine levels: To observe the effect of Phensedyl abuse on kidney function of the abusers, serum creatinine level of the addicts were determined and the results have been presented in Table 1. The results clearly indicated that no major changes in serum creatinine concentration occurred as a result of Phensedyl intake in addicts of all age groups and remained within the normal values (0.6-2.0 mg/dl).

Table 1. Serum creatinine concentration in Phensedyl abusers $(n=127)$.

\begin{tabular}{lcccc}
\hline \multirow{2}{*}{ Age group } & \multicolumn{2}{c}{ Normal value $(0.6-2 \mathrm{mg} / \mathrm{dl})$} & \multicolumn{2}{c}{ Raised value } \\
\cline { 2 - 5 } & Number & Percentage & Number & Percentage \\
\hline $18-25$ years & 12 & 9 & - & - \\
$26-35$ years & 101 & 80 & - & - \\
$35-55$ years & 14 & 11 & - & - \\
\hline
\end{tabular}

SGOT and SGPT levels: The SGOT activity of the Phensedyl addicts was measured to monitor for hepatic damages. The data gave evidence of no significant hepatic changes as the SGOT activity remained within the normal value in those who had been $(44 \%, n=56)$ taking CCS (Phensedyl) for less than 8 years. Similarly, SGOT levels of the abusers $(56 \%, n=71)$ who took the drug for more than 8 years also had normal values (Table 2).

Table 2. SGOT level of Phensedyl abusers depending on abuse time.

\begin{tabular}{lcclc}
\hline \multirow{2}{*}{ Period of abuse } & \multicolumn{2}{c}{ Normal value $(10-40 \mathrm{U} / \mathrm{l})$} & \multicolumn{2}{c}{ Raised value $(>40 \mathrm{U} / \mathrm{l})$} \\
\cline { 2 - 5 } & $\mathrm{n}$ & $\%$ & - & $\%$ \\
\hline$<8$ yrs. & 56 & 44 & - & - \\
$>8$ yrs. & 71 & 56 & - & - \\
Total & 127 & 100 & - & - \\
\hline
\end{tabular}

It was also observed that the serum SGOT level of the addicts remained within the normal range irrespective of the age group and the results have been presented in Table 3.

Table 3. Serum concentration of SGOT of phensedyl abusers $(n=127)$ \& non-addict controls $(n=50)$.

\begin{tabular}{ccccccccc}
\hline & \multicolumn{4}{c}{ Drug addict } & \multicolumn{3}{c}{ Non addict control } \\
\cline { 2 - 9 } Age group & \multicolumn{2}{c}{$\begin{array}{c}\text { Normal value (up to } \\
\text { 40 U/L) }\end{array}$} & \multicolumn{2}{c}{ Raised value } & \multicolumn{2}{c}{ Normal value } & \multicolumn{2}{c}{ Raised value } \\
\cline { 2 - 9 } & $\mathrm{n}$ & $\%$ & $\mathrm{n}$ & $\%$ & $\mathrm{n}$ & $\%$ & $\mathrm{n}$ & $\%$ \\
\hline $18-25$ years & 12 & 9 & - & - & 17 & 34 & - & - \\
$25-35$ years & 101 & 80 & - & - & 21 & 42 & - & - \\
$35-55$ years & 14 & 11 & - & - & 12 & 24 & - & - \\
\hline
\end{tabular}


SGPT or ALT is an enzyme present in higher concentration in liver than in muscle. Consequently an elevation is more specific for liver disease. The results of determination of serum SGPT activity of the present study has been presented in Table 4 .

Table 4. Serum SGPT level of Phensedyl addicts $(n=127)$ and non-addict controls $(n=50)$.

\begin{tabular}{rcccccccc}
\hline \multirow{2}{*}{ Age group } & \multicolumn{4}{c}{ Drug addict } & \multicolumn{3}{c}{ Non addict control } \\
\cline { 2 - 8 } & \multicolumn{2}{c}{$\begin{array}{c}\text { Normal value (upto } 40 \\
\text { U/L) }\end{array}$} & \multicolumn{2}{c}{ Raised value } & \multicolumn{2}{c}{ Normal value } & Raised value \\
\cline { 2 - 8 } $18-25$ years & 12 & 9.44 & - & - & 17 & 34 & - & - \\
$26-35$ years & 97 & 76.37 & 4 & 3.14 & 21 & 42 & - & - \\
$35-55$ years & 14 & 11.02 & - & - & 12 & 20 & - & - \\
\hline
\end{tabular}

The results suggested that codeine containing cough syrup had very little effect on the serum SGPT level of the Phensedyl abusers, as no significant changes in serum SGPT concentration was noticed. The number of individuals belonging to age group of $20-25$ years was 12 (9.44\%). Fourteen (14) of the abusers (11.02\%, age above 35 years) had the normal value of serum SGPT level (Table 4). However, 3.14\% $(n=4)$ of the abusers belonging to the age group of $26-35$ year had raised level of serum SGPT. On the other hand $76.37 \%(n=97)$ of abusers had normal value of same age group.

Serum Total cholesterol (TC) level: Serum TC of the addicts and non-addict controls are presented in Table 5. Results showed that TC level in the Phensedyl addict's sera was not significantly changed as a result of Phensedyl abuse.

Table 5. Serum total cholesterol (TC) concentration of Phensedyl abusers $(n=127)$ and non-addict controls $(n=50)$.

\begin{tabular}{|c|c|c|c|c|c|c|c|c|}
\hline \multirow{3}{*}{ Age group } & \multicolumn{4}{|c|}{ Drug addict } & \multicolumn{4}{|c|}{ Non addict control } \\
\hline & \multicolumn{2}{|c|}{$\begin{array}{l}\text { Normal value (100- } \\
240 \mathrm{mg} / \mathrm{dl})\end{array}$} & \multicolumn{2}{|c|}{ Raised value } & \multicolumn{2}{|c|}{$\begin{array}{c}\text { Normal value } \\
(100-200 \mathrm{mg} / \mathrm{dl})\end{array}$} & \multicolumn{2}{|c|}{ Raised value } \\
\hline & $\mathrm{n}$ & $\%$ & $\mathrm{n}$ & $\%$ & $\mathrm{n}$ & $\%$ & $\mathrm{n}$ & $\%$ \\
\hline $18-25$ years & 11 & 8.66 & - & - & 17 & 32 & - & - \\
\hline $26-35$ years & 102 & 80.31 & - & - & 21 & 42 & - & - \\
\hline $35-55$ years & 14 & 11.02 & - & - & 12 & 24 & - & - \\
\hline
\end{tabular}

Serum TC levels in the drug addicts ranged within 100 and $240 \mathrm{mg} / \mathrm{dl}$, while in the non-addict controls it was within $200 \mathrm{mg} / \mathrm{dl}$. But very interestingly, higher trends in the serum TC were observed in most of the phensedyl abusers and they had levels more than $200 \mathrm{mg} / \mathrm{dl}$ and abusing the drug for more than 8 years.

Serum Triglyceride (TG) level: Serum TG levels of the addicts and control subjects are presented in the Table 6. TG levels of addicts were significantly higher than the non-addict controls and the changes in serum TG 
values were found to be time-dependant. Out of total 127 addicts, $44.34 \%$ ( $n=56$ ) taking Phensedyl for less than 8 years had serum TG levels within the normal range (upto150 mg/dl). Among the addicts, $45.66 \%$ ( $n=$ 58) were recorded to have raised triglyceride values that appeared to be clinically very significant. The raised serum TG concentration observed in $n=56$ abusers who had been abusing Phensedyl for more than eight years was found to be statistically highly significant (shown in Anova table for Triglyceride, below Table 6).

In $10 \%$ of the addicts, who had abused Phensedyl for more than 8 year had normal serum triglyceride levels. ANOVA for triglyceride was calculated to be between groups SS value was 164037.6559, df was 1, MS was 164037.6559, $F$ was 115.59 and $P$ value was 0.0001 (ANOVA Table for triglyceride, below Table 6). In Pvalue interpretation values less than 0.01 is considered statistically very significant. In case of less than 8 years of abuse, the SD was $14.44, \mathrm{CV}$ was 13.54 and the t-value was 2.98. For more than 8 years, the SD and $\mathrm{CV}$ were 48.69, 27.20 and t-value was 9.82, which was more significant at $1 \%$ level (Table 8).

Table 6. Serum Triglyceride (TG) level in Phensedyl abusers $(n=127)$.

\begin{tabular}{|c|c|c|c|c|c|}
\hline \multirow[t]{2}{*}{ Time of abuse } & \multicolumn{2}{|c|}{ Normal value (50- $150 \mathrm{mg} / \mathrm{dl})$} & \multicolumn{3}{|c|}{ Raised value (above $150 \mathrm{mg} / \mathrm{dl}$ ) } \\
\hline & $\mathrm{n}$ & $\%$ & $\mathrm{n}$ & & $\%$ \\
\hline Below 8 yrs. & 56 & 44.34 & & & \\
\hline Above 8 yrs. & 13 & 10 & 58 & & 45.66 \\
\hline Total & 69 & 54.34 & 58 & & 45.66 \\
\hline \multicolumn{6}{|l|}{ ANOVA table } \\
\hline Source of Variance & SS & $d f$ & MS & $\mathrm{F}$ & P-value \\
\hline Between Groups & 164037.6559 & 1 & 164037.6559 & 115.59 & 0.0001 \\
\hline Within Groups & 177394.8008 & 125 & 1419.1584 & & \\
\hline Total & 341432.4567 & 126 & & & \\
\hline
\end{tabular}

HDL level: As shown in Tables 7-8 serum HDL levels of the addicts was significantly lower than the nonaddict controls. Of the addicts $53.53 \%$ had low level of HDL out of which $43.30 \%$ were taking Phensedyl for more than 8 years. Among the addicts, $46.38 \%$ were recorded to have normal value out of which $33 \%$ took the drug for less than 8 years. Lowering in HDL level noticed among addicts is clinically very significant and indicated that they might have developed various types of heart diseases. The decreased serum level of HDL was also found to be statistically highly significant in Analysis of variance (ANOVA for HDL, below Table 7). In P-value interpretation values less than 0.01 is considered statistically very significant. In our analysis between groups, the calculated $p$-value was 0.002 . Also the calculated t-value for more than 8 years of addiction was 12.69 (Table 8), which is significant at 1\% level, i.e. decrease in the HDL (good cholesterol) level than the normal value. Therefore, the reductions in $\mathrm{HDL}$ level were statistically very significant. 
Table 7. Serum HDL level in Phensedyl abusers $(n=127)$.

\begin{tabular}{|c|c|c|c|c|c|}
\hline \multirow[t]{2}{*}{ Time of abuse } & \multicolumn{2}{|c|}{ Normal value $(35-55 \mathrm{mg} / \mathrm{dl})$} & \multicolumn{3}{|c|}{ Low value (below $35 \mathrm{mg} / \mathrm{dl}$ ) } \\
\hline & $\mathrm{n}$ & $\%$ & $\mathrm{n}$ & & \\
\hline Below 8 yrs. & 42 & 33 & 13 & & \\
\hline Above 8 yrs. & 17 & 13.38 & 55 & & \\
\hline Total & 59 & 46.38 & 68 & & \\
\hline \multicolumn{6}{|l|}{ ANOVA table } \\
\hline Source of Variance & SS & df & MS & $\mathrm{F}$ & P-value \\
\hline Between Groups & 1129.4753 & 1 & 1129.4753 & 57.02 & 0.002 \\
\hline Within Groups & 2476.1078 & 125 & 19.8089 & & \\
\hline Total & 3605.5831 & 126 & & & \\
\hline
\end{tabular}

Table 8. Mean test for different characteristics of Triglyceride, HDL, LDL and non- addict controls

\begin{tabular}{|c|c|c|c|c|c|c|}
\hline & & $\mathrm{N}$ & Mean & SD & $\mathrm{CV}$ & t-value \\
\hline \multirow{3}{*}{ Control } & Triglyceride & 50 & 116.48 & 18.94 & 16.26 & \\
\hline & HDL & 50 & 42.33 & 4.29 & 10.14 & \\
\hline & $\mathrm{LDL}$ & 50 & 117.21 & 17.98 & 15.34 & \\
\hline \multirow{2}{*}{ Triglyceride } & $<8 \mathrm{yrs}$ & 56 & 106.64 & 14.44 & 13.54 & $2.98^{*}$ \\
\hline & $8+y r s$ & 71 & 179.03 & 48.69 & 27.20 & $9.82^{*}$ \\
\hline \multirow{2}{*}{$\mathrm{HDL}$} & $<8 \mathrm{yrs}$ & 55 & 39.07 & 5.49 & 14.06 & $3.40^{*}$ \\
\hline & $8+y r s$ & 72 & 33.05 & 3.45 & 10.45 & $12.69 *$ \\
\hline \multirow{2}{*}{ LDL } & $<8 \mathrm{yrs}$ & 55 & 114.39 & 16.38 & 14.32 & $0.84 \mathrm{~ns}$ \\
\hline & $8+y r s$ & 72 & 148.60 & 29.74 & 20.01 & $29.88^{*}$ \\
\hline
\end{tabular}

* = Significant at $1 \%$ level and ns = not significant, All t-values were compared with corresponding control value.

\section{LDL levels}

Serum LDL cholesterol level of the addicts are presented in the Tables 8 and 9 . Out of the total participants (127), 43.30\% ( $n=55$ ) who abused phensedyl for less than 8 years had LDL values within the normal ranges. 72 addicts' took Phensedyl (CCS) for more than 8 years and of them 43 addicts (34\%) had raised LDL cholesterol level which was clinically significant while the rest addicts had normal serum LDL levels.

In analysis of variance (ANOVA), the SS, df, MS, F and P-value between groups respectively were 36481.5232, 1, 36481.5232, 59.00 and 0.0001. In P-value interpretation values less than 0.01 is considered as statistically very significant (shown below Table 9). 
Table 9. Serum LDL level in Phensedyl abusers $(n=127)$.

\begin{tabular}{|c|c|c|c|c|c|}
\hline \multirow[t]{2}{*}{ Time of abuse } & \multicolumn{2}{|c|}{ Normal value (upto150 mg/dl) } & \multicolumn{3}{|c|}{ Raised value (above $150 \mathrm{mg} / \mathrm{dl}$ ) } \\
\hline & $\mathrm{n}$ & $\%$ & $\mathrm{n}$ & & $\%$ \\
\hline Below 8 yrs. & 55 & 43.30 & & & \\
\hline Above 8 yrs. & 29 & 22.83 & 43 & & 34 \\
\hline Total & 84 & 66 & 43 & & 34 \\
\hline \multicolumn{6}{|l|}{ ANOVA table } \\
\hline Source of Variance & SS & df & MS & $\mathrm{F}$ & P-value \\
\hline Between Groups & 36481.5232 & 1 & 36481.5232 & 59.00 & 0.0001 \\
\hline Within Groups & 77288.2450 & 125 & 618.3060 & & \\
\hline Total & 113769.7682 & 126 & & & \\
\hline
\end{tabular}

As shown in Table 8, the mean of addiction period less than 8 years were 114.39 and more than 8 years were 148.60. The SD in less than 8 years of intake was 16.38, whereas, more than 8 years of intake of Phensedyl was 29.74. The calculated t-value in below 8 years addict groups was 0.84 and found to be statistically non-significant. But the t-value calculated in case of addicts taking phensedyl above 8 years was 29.88 , which was significant at $1 \%$ level. The above statistical analyses indicated that the elevation in serum LDL cholesterol concentrations in phensedyl abusers were highly significant.

\section{Discussion}

In the present study, the serum creatinine levels of Phensedyl addicts remained unchanged even after long time abuse (8 years or more) of the drug. So, abuse of Phensedyl appears to have no major effect on renal function of the addicts. This finding of ours is similar to that reported by IPCS (International Program on chemical Safety) that codeine, promethazine and phenylephrine - the ingredients of Phensedyl syrup cause no major changes in renal function but may cause minor urinary retention in some people. But Jaffe and Kimmel (2006) in their recent critical review on Chronic Nephropathies of Cocaine and Heroin abuse mentioned that renal disease in cocaine and heroin abusers (in injectable form) has been associated with the nephrotic syndrome, acute glomerulonephritis, amyloidosis, interstitial nephritis, and rhabdomyolysis. As Phensedyl syrup is taken only by oral route while cocaine and heroin can be taken in different forms.

To evaluate the toxicity of Phensedyl on liver, SGOT and SGPT levels were measured from blood samples of the addicts. The SGOT and SGPT levels of the addicts remained within the normal values irrespective of age and drug abuse period of less or more than 8 years suggesting that Phensedyl abuse did not alter the liver function of the addicts.

Our such finding disagree with the findings of Balasubramaniam et al. (1994) working on Biochemical changes in Heroin (which is also an opiate as one of the ingredients of Phensedyl syrup) addicts reported SGOT and SGPT levels of heroin addicts (opiates) were significantly higher than in the non-addicts. Liver damage due to hepatitis is also reported by Abelson (1970) when heroin is regularly taken a few times a day in small doses. 
The results of lipid profile of the current study showed higher trends in the serum Total Cholesterol (TC) (>200 mg/dl), significantly elevated TG, raised LDL Cholesterol levels and significantly lower level of HDL Cholesterol levels in most of the Phensedyl (CCS) abusers abusing the drug for more than 8 years as compared to the non-addicts. The result suggests that Phensedyl alters the lipid profile causing dyslipidaemia after eight years of continuous intake of the drug i.e. the change in the lipid profile is time dependent.

The above finding of ours agrees very well with the study of Mohammadi et al. (2009) that opium use can increase serum levels of lipids that ends up as atheroma formation in the aorta of addicted rats. They also found out an association between the duration of addiction and increase in serum level of lipids, a risk factor, for cardiovascular diseases. Asgary et al. [18] showed that there was a direct correlation between opioids blood levels and duration of addiction. In their study, they also noted that the levels of Fibrinogen, apo B (Elevated levels of apo B correspond to elevated levels of LDL-C,) Lpa, were significantly higher in the case subjects as compared with controls, and that HDL-cholesterol and apo-a were significantly lower in the case subjects, That would mean that opium acts as a cardiovascular disease risk factor. Balasubramanium et al. (1994) working on Biochemical changes in Heroin (which is also an opiate drug like Phensedyl syrup) addicts that reported total serum, cholesterol to be significantly higher than the non addicts.

According to American Heart association cholesterol concentration more than $200 \mathrm{mg} / \mathrm{dl}$ raises the risk of stroke, coronary heart disease, ischemic stroke etc. Therefore, the Phensedyl abusers are at the risk of developing the above diseases.

A high level of $\mathrm{HDL}$ is an indication of a healthy metabolic system if there is no sign of liver disease or intoxication. HDL is called the "good cholesterol" because HDL cholesterol particles prevent atherosclerosis by extracting cholesterol from the artery walls and disposing of them through the liver. Thus, high levels of LDL cholesterol and low levels of HDL cholesterol (high LDL/HDL ratios) are risk factors for atherosclerosis, while low levels of LDL cholesterol and high level of HDL cholesterol (low LDL/HDL ratios) are desirable.

The decrease in the HDL level in the addicts is clinically significant. According to American Heart association low levels of serum $\mathrm{HDL}$ are associated with an increased incidence of $\mathrm{CHD}$ and increased mortality rate. At the other extreme, individuals with high concentrations of $\mathrm{HDL}$, as in familial hyperalphalipoproteinaemia seldom present with symptoms of $\mathrm{CHD}$ indicating a protective role of high concentrations of $\mathrm{HDL}$ against atherogenicity and development of CHD. LDL cholesterol is called "bad" cholesterol, as elevated LDL cholesterol is associated with an increased risk of coronary heart disease. LDL lipoprotein deposits cholesterol on the artery walls causing atherosclerosis. Lack of oxygen (ischemia) to the heart muscle causes chest pain, also formation of a blood clot in the artery can clause complete blockage of the artery, leading to death of heart muscle (heart attack). The results indicated that the Phensedyl abusers are at the risk of developing various types of heart diseases.

In conclusion, the results of the present study indicated that the Phensedyl abusers might develop various types of heart diseases like MI, Coronary Heart Disease CHD and TIA etc as there is the triad of: Elevated LDL cholesterol, Low HDL cholesterol and elevated Triglyceride.

\section{Acknowledgement}

The authors would like to extend thanks to the, Director of the Institute of Biological Sciences, University of Rajshahi, Bangladesh for providing financial support to purchase the necessary diagnostic kits and also providing the necessary laboratory facilities. 


\section{References}

Abelson PH. 1970. Death from heroin. Science.168, 1289. http://dx.doi.org/10.1126/science.168.3937.1289

Asgary S, Sarrafzadegan N, Naderi G, Rozbehani R. 2008. Effect of opium addiction on new and traditional ardiovascular risk factors: Do duration of addiction and route of administration matter? Lipids Health Dis 7, 42. http://dx.doi.org/10.1186/1476-511X-7-42

Battjes RJ, Pickens RW, Amsel Z. 1989. Introduction to HIV infection among intravenous drug abusers in low prevalence areas. J Acquire Immune Defic Syndr 2, 533-539.

CTC (Central drug addiction treatment center) 2000. Disease profile of the drug addicts. Prepared by Central Drug Addiction Treatment Centre, Tejgaon, Dhaka, Bangladesh (Unpublished).

Friedewald WT, Levy RI, Fredrickson DS. 1972. Estimation of the concentration of low density lipoprotein cholesterol in plasm without use of the preparative ultracentrifuge. Clin Chem, 18, 499-502.

Harris PD, Garret R.1972. Susceptibility of addicts to infection and neoplasia. N Eng J Med 287(6), 310. http://dx.doi.org/10.1056/NEJM197208102870620

ICDDR'B 1999. International Center for Diarrhoeal Diseases Research Bangladesh, National sentinel surveillance for HIV and syphilis, Dhaka, 1-11 pp.

Mohammadi A, Darabi M, Nasry M, Saabet-Jahromi JS, Afshar RM, Sheibani H. 2009 . Effect of opium addiction on lipid profile and atherosclerosis formation in hypercholesterolemic rabbits. Experim Toxic Pathol 61, 145-149. http://dx.doi.org/10.1016/i.etp.2008.08.001

Release of INCB Report for 1995. Regional Update: South Asia. Available from: $<$ http://www.un.org/ecosocdev/geninfo/drugs/souasi_e.htm)> [8 February 2010].

Two held with Phensedyl in Gaibandha. The Daily Sun, DHAKA, Sunday 05 December, 2010). Available at: $<h$ ttp://www.daily-sun.com/details_yes_05-12-2010_two-held-with-phensidyl-in-gaibandha_58_1_8_1_3.html> [Accessed on: 10 Oct 2011].

UNDCP 1997. The health and social consequence of drug abuse. In: World Drug Report, UNDCP, Oxford University Press, New York, 70-121 pp.

Varela P, Marcos A, Ripoll S, Santacruz I, Requejo AM. 1997. Effects of HIV infection and detoxification time on anthropometric measurements and dietary intake of male drug addicts. Am J Clin Nut 66(Suppl), 509-514.

WHO.1992. Programme on substance abuse, ATLAS report, WHO, 1-75 pp.

Jaffe JA, Kimmel PL. 2006 (July). Chronic nephropathies of cocaine and heroin abuse: a critical review. Clin J Am Soc Nephrol 1(4), 655-667. http://dx.doi.org/10.2215/CJN.00300106

Balasubramaniam K, Vasanthy Arasaratnum, Samanthy Nadarajah, Mary Sorubiny Chelliah, Laksman P, Arulampalam PK. 1994. Biochemical changes in heroin addicts. J Natn Sci Coun Sri Lanka 22 (1), 1-5,

IPCS (International Program on chemical Safety) http://www.inchem.org/ ) 